\title{
Regulatory role of miRNA-23a in diabetic retinopathy
}

\author{
LIHUI SUN ${ }^{1,2}$, XUEZHENG LIU ${ }^{1,3}$ and ZHONGFU ZUO ${ }^{3,4}$ \\ ${ }^{1}$ Department of Anatomy, Histology and Embryology, Guangxi Medical University, Nanning, \\ Guangxi Zhuang Autonomous Region 530021; ${ }^{2}$ The Fifth Department of General Surgery, \\ The Third Affiliated Hospital of Jinzhou Medical University; ${ }^{3}$ Liaoning Key Laboratory of \\ Diabetic Cognitive and Perceptive Dysfunction, Department of Anatomy, Histology and Embryology, \\ Jinzhou Medical University, Jinzhou, Liaoning 121000; ${ }^{4}$ Department of Anatomy, Histology and Embryology, \\ Postdoctoral Research Station, Guangxi Medical University, Nanning, \\ Guangxi Zhuang Autonomous Region 530021, P.R. China
}

Received March 9, 2020; Accepted March 12, 2021

DOI: $10.3892 /$ etm.2021.10912

\begin{abstract}
The present study aimed to investigate the expression of microRNA (miRNA)-23a in blood and tear samples from diabetic retinopathy (DR) patients. Blood and tear samples were obtained from 33 patients with proliferative DR. Additionally, a rat model of DR was established. Reverse transcription-quantitative PCR was used to determine vascular endothelial growth factor (VEGF) mRNA and miRNA-23a expression levels, while ELISA and western blot analysis were performed to determine protein expression levels. Bioinformatics analysis and dual luciferase reporter assay were used to predict and validate the interaction between miRNA-23a and VEGF and cell proliferative ability was assessed with the MTT assay. In comparison to control patients VEGF mRNA and protein expression levels were significantly elevated in the blood and tear samples from patients with DR, while the expression level of miRNA-23a was significantly reduced. In blood and retinal tissues from a rat model of DR, the mRNA and protein expression levels of VEGF were significantly increased, while the miRNA-23a expression level was significantly decreased relative to controls. Dual luciferase reporter assay showed that miRNA-23a bound to the 3'-untranslated region (UTR) of VEGF. Moreover, over-expression of miRNA-23a significantly reduced the expression level of VEGF and the proliferative activity of human retinal microvascular endothelial cells. The elevated VEGF expression in the blood and tears of patients with DR may be related to the reduced miRNA-23a
\end{abstract}

Correspondence to: Dr Xuezheng Liu, Liaoning Key Laboratory of Diabetic Cognitive and Perceptive Dysfunction, Department of Anatomy, Histology and Embryology, Jinzhou Medical University, 3-40 Songpo Road, Jinzhou, Liaoning 121000, P.R. China

E-mail: liuxuezhenga168@163.com

Key words: diabetic retinopathy, miRNA-23a, vascular endothelial growth factor expression. miRNA-23a may regulate microvascular growth at the retina via VEGF and contribute to DR progression.

\section{Introduction}

Diabetic retinopathy (DR) is a microvascular complication associated with diabetes mellitus (DM) that is usually caused by hyperglycemia-induced leakage of the retinal capillary wall (1). Diabetes can cause various physiological and pathological changes to eye structure and function, including cataracts, glaucoma and optic nerve atrophy (2). Pathophysiological changes in the retina are important causes of visual function loss in DM patients (3). According to the results of previous studies, impaired physiological function of the retina in patients with DM is most often caused by blockage of the microcirculation (4-7). It has been established that altered microvessel structure, as is the case where increased basement membrane thickness reduces the inner diameter of the blood vessel, represents one of the causes of microcirculation blockage (8). Retinopathy is often detected in patients with DM and the risk of these patients developing proliferative diabetic retinopathy (PDR)-induced vision loss is increased (9), which should be distinguished from DR, the generalized diseases.

Clinical studies have indicated that there is an elevated concentration of various inflammatory cytokines in the serum and vitreous humor of patients with PDR, including monocyte chemotactic protein-1, interleukin (IL)-1 $\alpha$, IL-6, tumor necrosis factor- $\alpha$ and vascular endothelial growth factor (VEGF) $(10,11)$. There have also been reports investigating the roles of VEGF in DR (12-14). Jain et al (15) have suggested that high expression levels of VEGF and intercellular adhesion molecule-1 in DM patients are associated with destruction of the retinal outer membrane and the internal and external photoreceptor segments, which is positively correlated with the degree of retinopathy. As an important risk factor for promoting PDR neovascularization (thus enhancing irregular growth of blood vessels and increasing microcirculation burden) (16,17), VEGF can not only directly bind to vascular endothelial cells to promote pathological proliferation, but 
also induce the secretion of other inflammatory factors, contributing to disease progression (18). To the best of our knowledge, the mechanism underlying VEGF regulation has not been fully elucidated.

MicroRNAs (miRNAs) are a class of 18-22-nucleotide non-coding small RNA molecules in eukaryotes, which regulate gene expression at the mRNA level $(19,20)$. Altered expression levels of multiple miRNAs and proteins have been observed in the pathogenesis of DR, suggesting that miRNAs may play important roles in regulating the levels of genes and proteins in disease pathogenesis $(21,22)$. miRNA-23a has been indicated to be involved in angiogenesis in lung cancers and nasopharyngeal carcinomas $(23,24)$. In cardiovascular diseases, such as the coronary heart disease, miRNA-23a inhibits VEGF expression and regulates angiogenic processes (25). The regulation of VEGFA by microRNA is closely related to angiogenesis. For example, miRNA-134 has been suggested to inhibit angiogenesis and osteosarcoma proliferation through targeting the VEGFA/VEGFR1 pathway, and miRNA-29b inhibits the angiogenesis of endometrial cancer by targeting VEGFA $(26,27)$. However, to the best of our knowledge, there have been no reports concerning the effects of miRNA-23a on VEGF in the pathogenesis of DR.

In the present study, the roles of miRNA-23a and VEGF in the pathogenesis of DR were investigated. The expression levels of VEGF and miRNA-23a in blood and tear samples from patients with PDR and in a rat DR model were determined and analyzed. The relationship and interaction between miRNA-23a and VEGF were also investigated and discussed.

\section{Materials and methods}

Study subjects. In total 33 patients with proliferative type 2 DR (PDR), 15 males and 18 females with a median age of 54.6 years old (age range, 38 to 62 years), who were admitted to The Third Affiliated Hospital of Jinzhou Medical University (Liaoning, China) between June and December 2013, were included in this study. A further 28 healthy subjects, 13 males and 15 females, with a median age of 55.2 years old (age range, 39-63), were also included as the control group during the same period and from the same hospital. Blood and tear samples were collected from these subjects. All patients were diagnosed by the same fundus specialist, based on dilated fundus examination and photography, as well as binocular B-ultrasound. Inclusion criteria for patients with PDR was as follows: i) Subject met the WHO diagnostic criteria for type 2 diabetes 1999 (28); and ii) patients whose findings from the fluorescein fundus angiography under fully dilated condition met the diagnostic criteria for PDR according to the DR diagnosis and treatment 2014 guidelines from the Chinese Fundus Group (29). Among the PDR patients, 8 cases were at the early stage of hyperplasia, 20 cases at the fibrous hyperplasia-stage and 5 cases at the late hyperplasia stage. All patients were treated in accordance with Chinese PDR treatment guidelines. In the control group, diabetes and retinopathy were clearly excluded according to the examination. The study was approved by The Ethics Committee of The Third Affiliated Hospital of
Jinzhou Medical University, and written informed consent was obtained from each subject.

Sample preparation. For the collection of peripheral blood serum, density gradient centrifugation combined with the adherent separation was conducted. Peripheral venous blood samples (10-15 ml; anti-coagulated with EDTA, $1.5 \mathrm{mg} / \mathrm{ml})$ were obtained from each subject and placed at $4^{\circ} \mathrm{C}$ for $1-2 \mathrm{~h}$. The upper supernatant in the tube was collected and centrifuged at $400 \mathrm{x}$ g for $10 \mathrm{~min}$ and then stored at $-70^{\circ} \mathrm{C}$. The tear samples were harvested from the patients and healthy subjects on the day of the physical examination and stored at $-70^{\circ} \mathrm{C}$. Before sampling, the skin of the lower eyelid was smeared with alcohol. When the reflective tears appeared, a capillary was used to suck the tears from the outer canthus.

Study animals and model establishment. A total of 30 male specific pathogen free Sprague-Dawley rats, 50-days-old, weighing 180-200 g, were provided by Chongqing Tengxinbier Experimental Animal Co., Ltd. [animal approval number: SCXK (Yu) 2016-0012; Chongqing, China]. Before experiments, all rats were acclimatized for one week, in a 12-h light/dark cycle, at the constant temperature of $24 \pm 2^{\circ} \mathrm{C}$, with a humidity of $55 \pm 5 \%$, and free access to food and water. The $3 \mathrm{R}$ animal welfare principle (30) was followed during the animal experiments.

For the model establishment, after fasting for $12 \mathrm{~h}$, the SD rats were intraperitoneally injected with streptozotocin (STZ; Sigma-Aldrich; Merck KGaA; in 0.1 mol/l sodium citrate buffer, $\mathrm{pH} 4.6$ ), at $60 \mathrm{mg} / \mathrm{kg}$ body weight. Rats in the control group were injected with an equivalent volume of sodium citrate buffer. After $72 \mathrm{~h}$, venous blood samples were collected through the tail vein, for random blood glucose monitoring. Model establishment was considered to be successful when the random blood glucose level exceeded the level of $16.7 \mathrm{mmol} / \mathrm{l}$, which was maintained for one week. After successful model establishment, animal body weights and the random blood glucose levels were recorded every week (31). Before sacrifice, animals were injected with $50 \mathrm{mg} / \mathrm{kg}$ body pentobarbital and then subject to the decapitation. All animal experiment were performed in accordance with the guidelines of the Ethics Committee of Guangxi Medical University.

Reverse transcription-quantitative-PCR (RT- $q P C R)$. Total RNA was extracted from the hRMECs with TRIzol ${ }^{\circledR}$ (Invitrogen; Thermo Fisher Scientific, Inc.). cDNA was obtained from the total RNA with reverse-transcription with the TIANScript II cDNA First Strand Synthesis kit (cat. no. KR107; Tiangen Biotech Co., Ltd.) at $42^{\circ} \mathrm{C}$. The qPCR was performed with the miRcute miRNA Fluorescence Quantification kit (cat. no. FP401; Tiangen Biotech. Co., Ltd.) with the SuperReal PreMix (SYBR Green) (cat. no. FP204; Tiangen Biotech. Co., Ltd.) on the iQ5 qRT-PCR instrument (Bio-Rad, Laboratories, Inc.).

For the determination of human VEGF mRNA levels, the following primer sequences were used: human VEGF, forward 5'-TTGCCTTGCTGCTCTACCTC-3' and reverse 5'-AAATGCTTTCTCCGCTCTGA-3'; and human $\beta$-actin, forward 5'-TGACGTGGACATCCGCAAAG-3' and reverse 5'-CTGGAAGGTGGACAGCGAGG-3'. The 25- $\mu 1$ system 
consisted of $12.5 \mu \mathrm{l}$ SYBR Premix EXTaqTM, $10 \mu \mathrm{M}$ primer

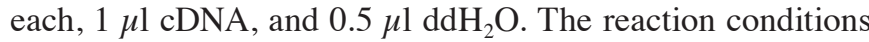
were as follows: $94^{\circ} \mathrm{C}$ for $2 \mathrm{~min}$, followed by 34 cycles of $94^{\circ} \mathrm{C}$ for $30 \mathrm{sec}, 55^{\circ} \mathrm{C}$ for $30 \mathrm{sec}$ and $71^{\circ} \mathrm{C}$ for $1 \mathrm{~min}$ followed by a final extension at $71^{\circ} \mathrm{C}$ for $2 \mathrm{~min}$.

For the determination of rat VEGF mRNA expression levels, the following primer sequences were used: miRNA-23a, forward 5'-CCATGAACTTTCTGCTCTTC-3' and reverse 5'-GGTGAGAGGTCTAGTTCCCGA-3'; and GAPDH, forward 5'-CCCTCAATGACCACTTTGTG-3' and reverse 5'-GGTTTGAGGGCTCTTACTCCT-3'. The reaction conditions were set as follows: $95^{\circ} \mathrm{C}$ for $10 \mathrm{~min}$ followed by 40 cycles of $95^{\circ} \mathrm{C}$ for $30 \mathrm{sec}, 55^{\circ} \mathrm{C}$ for $20 \mathrm{sec}$ and $72^{\circ} \mathrm{C}$ for $30 \mathrm{sec}$.

For the determination of miRNA-23a expression levels in the tissues, the following primer sequences were used: miRNA-23a, forward 5'-ATCACATTGCCAGGGA-3' and reverse 5'-CAGTGCGTGTCGTGGAGT-3'; and U6, forward 5'-CTCGCTTCGGCAGCACA-3' and reverse 5'-AACGCT TCACGAATTTGCGT-3'. The reaction conditions were set as follows: $95^{\circ} \mathrm{C}$ for $5 \mathrm{~min}$ followed by 40 cycles of $95^{\circ} \mathrm{C}$ for $10 \mathrm{sec}$, $60^{\circ} \mathrm{C}$ for $35 \mathrm{sec}$ and 72 for $30 \mathrm{sec}$. Relative expression levels of the target genes were calculated with the $2^{-\Delta \Delta C q}$ method (32). $\beta$-actin, GAPDH and U6 were used as internal references.

Western blot analysis. Tissue samples and cells were lysed with the RIPA lysis solution (cat. no. P0013B; Beyotime Institute of Biotechnology). Protein concentration was determined with the BCA method (cat. no. RTP7102; ZhongKeRuitai Biotechnology Co, Ltd.). A total of $20 \mu \mathrm{g}$ of each protein was separated by $10 \%$ SDS-PAGE and then transferred onto the PDVF membrane. After blocking with 5\% non-fat milk at room temperature for $1 \mathrm{~h}$, the membrane was incubated with rabbit anti-human anti-VEGF polyclonal primary antibody (1:1,000 dilution; cat. no. ab46154; Abcam) or anti- $\beta$-actin primary antibody (1:5,000 dilution; cat. no. ab129348; Abcam) at $4^{\circ} \mathrm{C}$ overnight. The membrane was then incubated with goat anti-rabbit secondary antibody (1:3,000 dilution; cat. no. ab6721; Abcam) at room temperature for $1 \mathrm{~h}$. Blots were visualized using ECL reagent (cat. no. ab65623; Abcam). Protein bands was acquired and analyzed using Image lab 3.0 software (Bio-Rad Labratories, Inc.). $\beta$-actin was used as internal reference.

Enzyme-linked immunosorbent assay (ELISA). Blood samples were centrifuged at $1,000 \mathrm{x} \mathrm{g}$, at $4^{\circ} \mathrm{C}$, for $10 \mathrm{~min}$ to obtain the serum, and the tear samples were directly harvested. The VEGF levels were determined with ELISA kits (Human VEGF ELISA kit; cat. no. ab222510; and Rat VEGF ELISA kit; cat. no. ab100786; Abcam). According to the manufacturer's instructions, $50 \mu \mathrm{l}$ standard samples at indicated concentrations were added into the standard wells, while $10 \mu 1$ serum or tear samples were added into the sample wells, followed by $40 \mu \mathrm{l}$ of diluting solution. HRP-labeled detection antibody $(100 \mu 1)$ was added into the standard and sample wells. The plate was sealed and incubated for $1 \mathrm{~h}$. After washing for 5 times, substrates A and B (50 $\mu \mathrm{l}$ each) were added into the wells and the plate was incubated at $37^{\circ} \mathrm{C}$ for $15 \mathrm{~min}$. A total of $50 \mu \mathrm{l}$ of stopping solution was added into each well and the OD values at $450 \mathrm{~nm}$ were read within 15 min.
Bioinformatics analysis. Based on retrieved references (33-36), bioinformatics analysis was performed to predict the up-stream miRNA that would interact with VEGF, to further investigate the regulatory mechanism underlying the DR pathogenesis. miRanda software (2010 version; http://www.microma. org/rnicroma/home.do) was used for the gene prediction, according to the online software operating instructions.

Cell transfection. For cell transfection, the hRMECs or 293 cells were seeded onto 24-well plates at the density of $3 \times 10^{5}$ cells/well with F12/DMEM medium containing $10 \%$ FBS without antibiotics, which were incubated at $37^{\circ} \mathrm{C}$. The cell culture duration varied from cell to cell, to achieve the cell growth confluence of $70 \%$, which was generally 24-48 h. Cell transfection was performed when $70 \%$ confluence was achieved. The plasmid/siRNA/agomiR (designed and synthesized by Sangon Biotech. Co., Ltd.) sequences were: AgomiR-23a, forward 5'-GGGGUUCCUGGG GAUGGGAUUU-3', and reverse, 5'-AAAUCCCAUCCCCAG GAACCCC-3'; agomiR-NC forward, 5'-UUUGUACUACAC AAAAGUACUG-3', and reverse, 5'-CAGUACUUUUGUGUA GUACAAA-3'). Plasmid $(0.8 \mu \mathrm{g}) / \mathrm{siRNA}(100 \mathrm{nM}) /$ agomiR $(100 \mathrm{nM})$ and lipofectamine ${ }^{\circledR} 2000(1 \mu \mathrm{l})$ were added to the EP tubes containing $50 \mu \mathrm{l}$ Opti Memi medium (Thermo Fisher Scientific, Inc.), respectively. After $5 \mathrm{~min}$, the solution in these two tubes were mixed together. After a further $20 \mathrm{~min}$ at room temperature, the mixture was used to incubate the cells for $6 \mathrm{~h}$ and then the medium was replaced with F12/DMEM medium (Thermo Fisher Scientific, Inc.). After 48 h, the cells were collected for analysis.

Dual luciferase reporter assay. The wild-type and mutant sequence regions in the VEGF 3'-untranslated region (UTR) for miRNA-23a were chemically synthesized by the Sangon, Biotech Co., Ltd. with the Spe-1 and HindIII restriction sites at each end, respectively. These two DNA fragments were cloned into the pMIR-REPORT luciferase reporter plasmid from the Dual-Luciferase ${ }^{\circledast}$ Reporter Assay System (Promega Corporation), and the mutant 3 '-UTR region was used as a control. The sequences were as follows: hsa-miR-23a, 3'-CCU UUAGGGACCGUUACACUA-5'; VEGFA, 5'-AUGUUUAUG UAUAUAUGUGAU-3'; rno-miR-23a, 3'-CCUUUAGGGACC G-UUACACUA-5'; and Vegfa, 5'-CCUGCAGCAUAGCAG AUGUGAA-3'.

The plasmids $(0.8 \mu \mathrm{g})$ containing the wild-type and mutant 3'-UTR DNA sequences were transfected into 293T cells (Cell Bank of Type Culture Collection of The Chinese Academy of Sciences) using Lipofectamine ${ }^{\circledR}$ for $24 \mathrm{~h}$ in a $37^{\circ} \mathrm{C}, 5 \% \mathrm{CO}_{2}$ incubator, in 12/DMEM medium. Then $100 \mathrm{nM}$ agomiRNA-23a was transfected into the cells for $24 \mathrm{~h}$. The cells were then lysed, and the luciferase was detected using a GloMax 20/20 sluminometer. Renilla was used as internal reference.

MTT assay. hRMECs (human retinal microvascular endothelial cells) were purchased from the Cell Bank of Type Culture Collection of The Chinese Academy of Sciences and were seeded onto 96-well plates at a density of $2 \times 10^{3} /$ well. At 24,48 , and $72 \mathrm{~h}$, respectively, $20 \mu 1$ MTT $(5 \mathrm{~g} / 1)$ was added into each well. At each indicated time point, MTT was added 
into the wells and incubated at $37^{\circ} \mathrm{C}$ for $4 \mathrm{~h}$, and then $150 \mu \mathrm{l}$ of DMSO was added. The absorbance at $490 \mathrm{~nm}$ was detected, and the cell proliferation curve was plotted accordingly.

Statistical analysis. Data are presented as the mean \pm SD. SPSS 18.0 (SPSS, Inc.) was used for statistical analysis. After the normality test, the one-way ANOVA was used for multiple group comparison, with the LSD and SNK tests for data with homogeneous variance, or the Tamhane's T2 or Dunnett's T3 test for data with non-homogeneous variance. $\mathrm{P}<0.05$ was considered statistically significant.

\section{Results}

VEGF mRNA and protein expression levels in human samples and rat model samples. To determine the mRNA and protein expression levels of VEGF in the human serum and tear samples, RT-qPCR and ELISA were performed. The results indicated that in comparison with the control group, both the mRNA and protein expression levels of VEGF in the human serum and tear samples were significantly increased in patients with DR (both $\mathrm{P}<0.05$; Fig. 1).

mRNA and protein expression levels of VEGF in the serum and retina samples of diabetic rat models were determined by RT-qPCR, ELISA and western blot analysis. The results showed that in comparison with the control group, the mRNA and protein expression levels of VEGF in the serum and retina of diabetic rats was significantly increased (both $\mathrm{P}<0.05$; Fig. 1). Taken together, these results suggest that VEGF may play a regulatory role in the disease pathogenesis of DR.

Expression of miRNA-23a in human samples and rat model samples. To determine the miRNA-23a expression levels in the human serum and tear samples RT-qPCR was performed. The results showed that in comparison with the control group, miRNA-23a expression levels were significantly reduced in the human serum and tear samples in the patients with DR ( $\mathrm{P}<0.05$; Fig. 2).

miRNA-23a expression levels in the serum and retina samples of diabetic rats were determined by RT-qPCR. The results indicated that in comparison with the control group, the miRNA-23a expression levels in the serum and retina samples of diabetic rats were significantly reduced $(\mathrm{P}<0.05$; Fig. 2). Taken together, these results suggest that miRNA-23a may be involved in the pathogenesis and development of DR.

Prediction and confirmation of interaction between miRNA-23a and VEGF. miRanda software (http://www. microma.org/rnicroma/home.do) was used to predict the possible VEGF regulatory genes. The results showed that miRNA-23a could be a probable regulatory gene (Fig. 3). Dual luciferase reporter assay was performed to confirm the interaction between the miRNA-23a and VEGF. The results indicated that after co-transfection with the agomiRNA-23a and pMIR-REPORT luciferase reporter plasmids, the luciferase values were significantly reduced $(\mathrm{P}<0.05)$, while there were no significant changes in the mutant group (P>0.05; Fig. 3). These results suggested that miRNA-23a binds to the 3'-UTR of VEGF to regulate the gene expression.

Effects of agomiRNA-23a transfection on hRMECs. hRMECs were transfected with agomiRNA-23a and cell proliferative ability was assessed with the MTT assay. The results showed that after transfection the VEGF expression levels in the hRMECs were significantly down-regulated. Moreover, after transfection with agomiRNA-23a, the cell proliferation ability was significantly declined $(\mathrm{P}<0.05)$ (Fig. 4). These results suggest that agomiRNA-23a would influence the VEGF to inhibit the proliferation ability of hRMECs.

\section{Discussion}

In the present study the mRNA and protein expression levels of VEGF in the serum and tear samples of patients with DR were determined as were the expression levels of its upstream regulatory gene miRNA-23a. Moreover, a rat model of diabetes was established and the expression levels of VEGF and miRNA-23a in the blood and retina tissues of diabetic rats were detected. Direct binding between miRNA-23a and VEGF, as well as related biological functions, were preliminarily explored and the molecular mechanism underlying the regulatory effects of miRNA-23a on VEGF in the pathogenesis of DR were studied.

DR is a multifactorial chronic progressive disease, which is characterized by altered retinal microvascular blood flow, impaired function and apoptosis of pericytes and endothelial cells and increased vascular permeability, further aggravating the occlusion of capillary vessels (37). The formation of non-perfused areas results in neovascularization, leading to repeated retinal hemorrhage and fibroproliferation, recognized as the proliferative diabetic retinopathy (PDR) (38). With the progression of DR, the degrees of ischemia and hypoxia are further aggravated, which may increase the secretion of various growth-promoting factors (such as fibroblast growth factor 21 and VEGF) in the vitreous cavity, further leading to the imbalance between the intraocular angiogenesis and inhibitory factors, and promoting the disease into the proliferative phase, ending with the development of refractory neovascular glaucoma (39). The typical pathological features of PDR include neovascularization and fibroproliferation, which are also the most important pathological factors leading to visual loss in patients with DR (40). Persistent inflammatory responses and up-regulated expression levels of angiogenic factors are closely related to the pathogenesis of PDR. Cytokines such as VEGF, interleukin and growth factors play important roles in pathological process (41). As a potent angiogenic agent, VEGF is a core factor in PDR development. The biological activity of VEGF is closely related to its expression level. In normal retinal tissues, VEGF is expressed at low levels, regulating physiological angiogenesis (18) and exerting neuroprotective effects (41). Jain et al (15) found that in comparison with normal healthy people, the serum VEGF levels in diabetic patients were significantly increased along with the disease progression, in the following order: $\mathrm{PDR}>\mathrm{NPDR}>\mathrm{DM}$ with no retinopathy $>$ healthy people. Moreover, the VEGF expression levels in the aqueous humor and vitreous humor are associated with retinopathy in patients with DM (42). In the present study the VEGF expression levels in the serum 
A

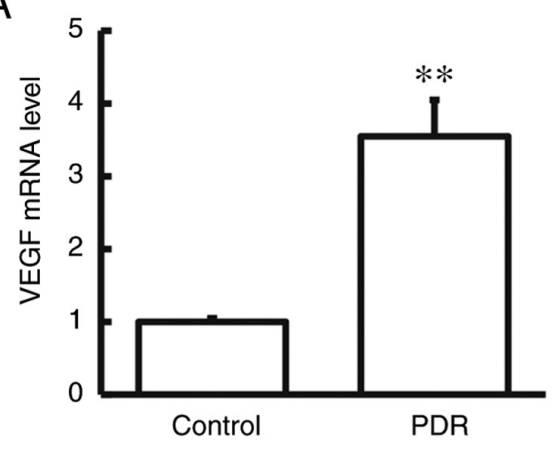

C

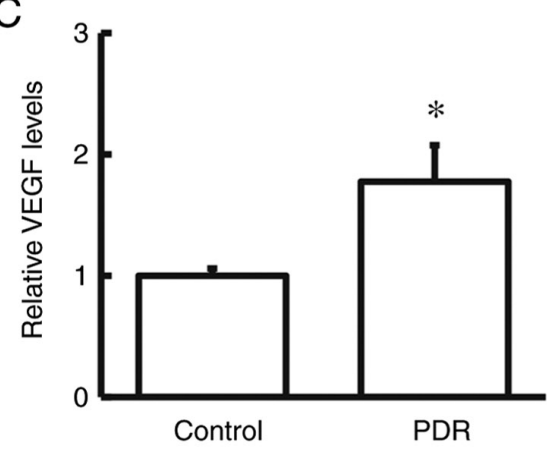

E

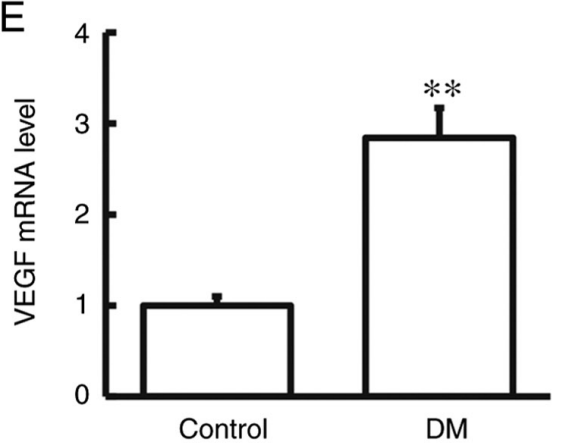

G

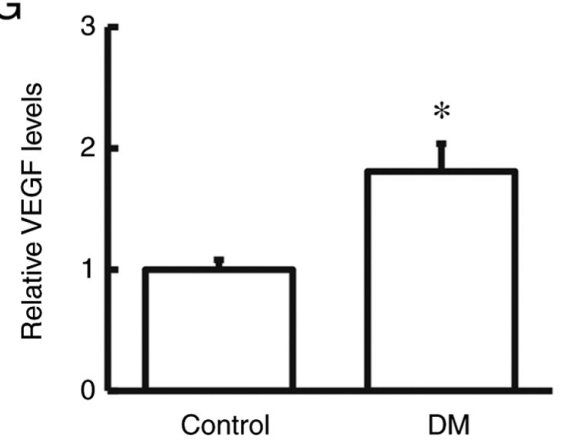

B
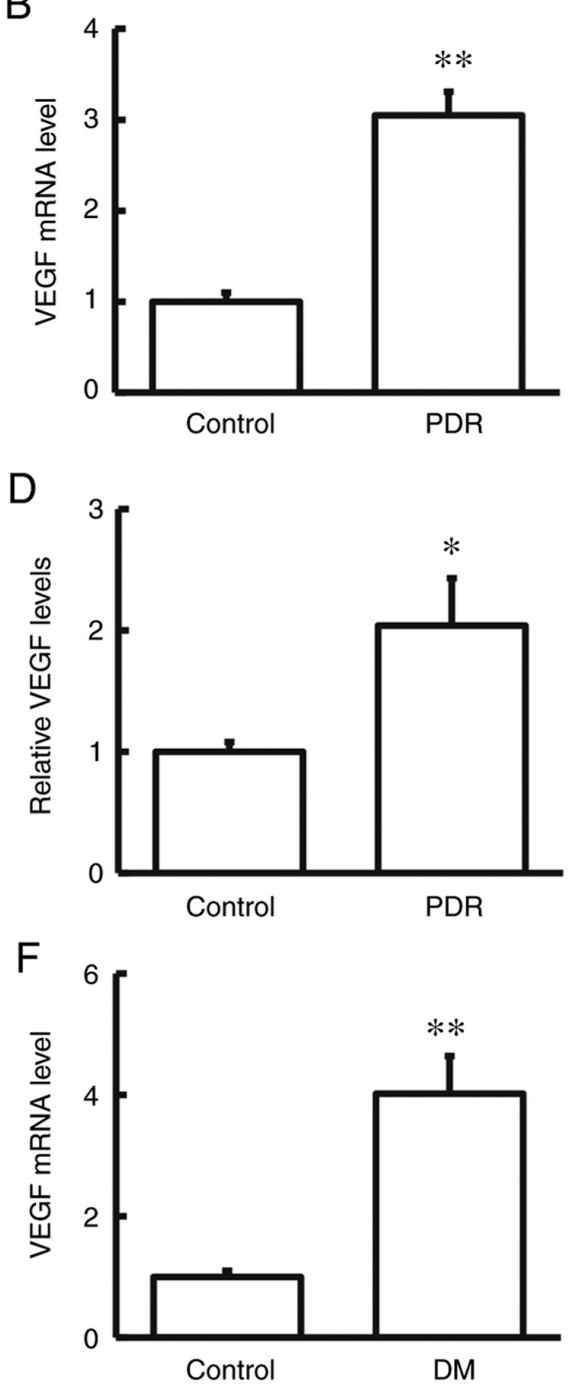

$\mathrm{H}$

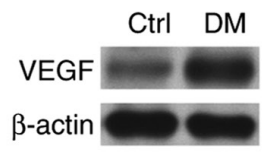

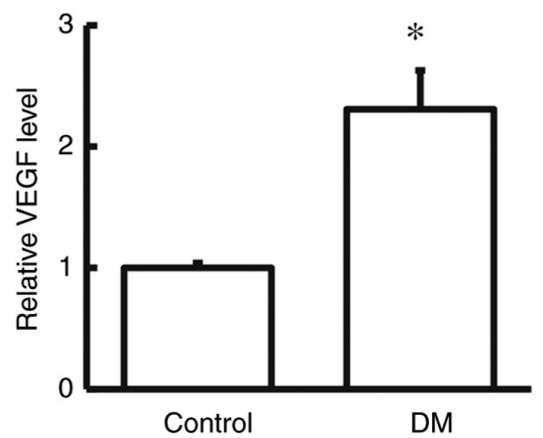

Figure 1. Analyses of VEGF mRNA and protein expression levels. VEGF mRNA expression levels in the (A) serum and (B) tear samples from the PDR were assessed using RT-qPCR. The VEGF concentration in the (C) serum and (D) tear samples from the PDR were determined by ELISA. The VEGF mRNA expression levels in the (E) serum and $(\mathrm{F})$ retina tissues from the rat DM were determined with RT-qPCR. The VEGF concentration in the $(\mathrm{G})$ serum and $(\mathrm{H})$ retina tissues from the rat DM were determined with ELISA and western blot analysis, respectively. ${ }^{*} \mathrm{P}<0.05,{ }^{* *} \mathrm{P}<0.01$ vs. control. VEGF, vascular endothelial growth factor; RT-qPCR, reverse transcription quantitative PCR; Ctrl, control; PDR, patients with diabetic retinopathy; DM, diabetic model.

and tear samples from the patients with DR were significantly increased in comparison with healthy individuals. In line with this, results from the rat diabetic models showed that, the VEGF expression levels in the serum and retina tissues were increased in comparison with normal rats. VEGF and angiogenesis are closely related, and angiogenesis has been proven to be an important feature for the progression of PDR (43). Therefore, VEGF was a focus of the present investigation. Taken together, the results of the present study suggest an important role for VEGF in the pathogenesis and development of DR.

The mechanism underlying the regulatory effects of VEGF has not been fully elucidated. A correlation between VEGF and miR-23 has been previously reported (44). In the present study, results from bioinformatics prediction indicated that miRNA-23a was an upstream miRNA regulating VEGF, with similar and highly conservative sequences within the 
A

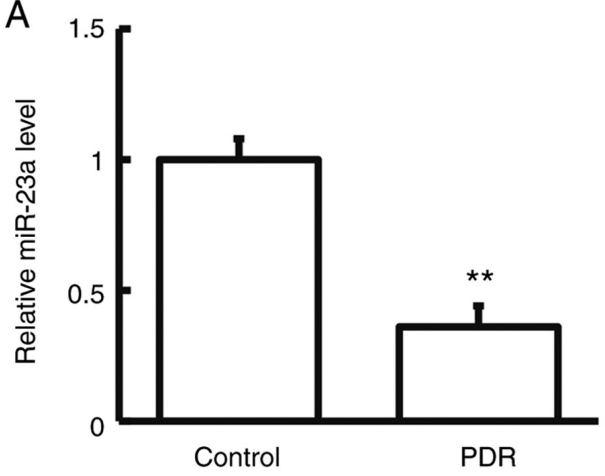

C

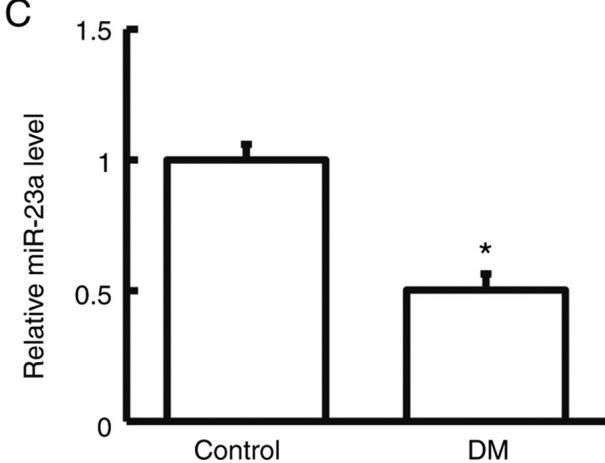

B

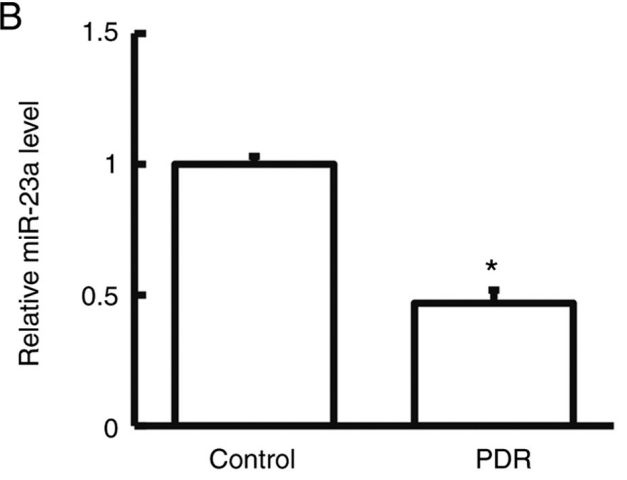

D

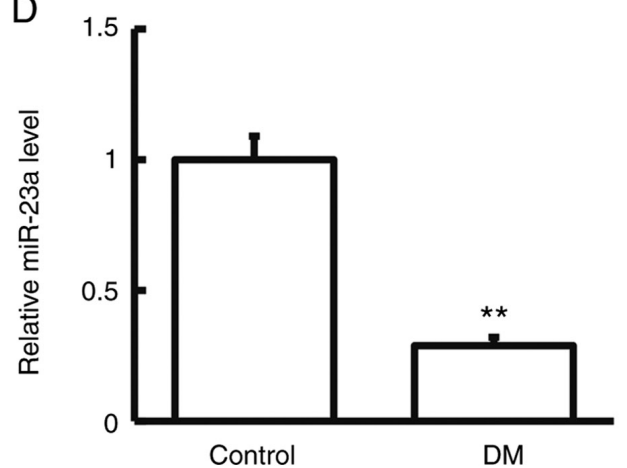

Figure 2. Analyses of miRNA-23a expression levels. The miRNA-23a expression levels in the (A) serum and (B) tear samples from the PDR were determined with RT-qPCR. The miRNA-23a expression levels in the (C) serum and (D) retina tissues from the rat DM were determined with RT-qPCR. "P<0.05, ${ }^{* *} \mathrm{P}<0.01$ vs. control. miR, microRNA; PDR, patients with diabetic retinopathy; RT-qPCR, reverse transcription quantitative PCR; DM, diabetic model.

A

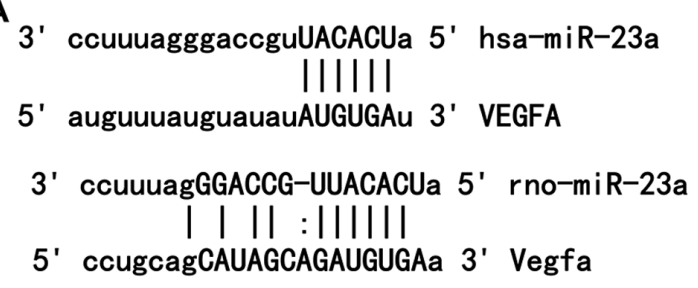

B

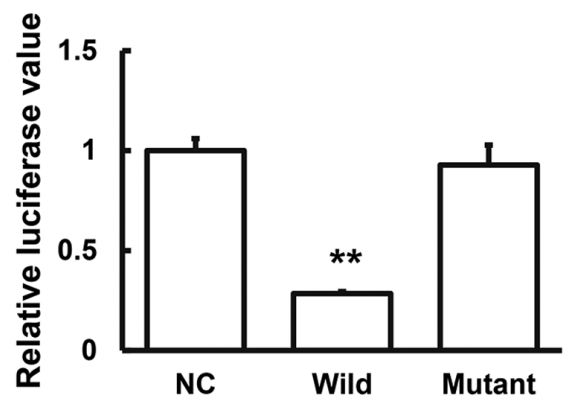

Figure 3. Bioinformatics prediction of VEGF regulation. (A) Bioinformatics prediction of up-stream regulating gene of VEGF. (B) Dual luciferase reporter assay, the $293 \mathrm{~T}$ cells were co-transfected with the agomiRNA-23a and pmiR-REPORT luciferase reporter plasmids containing the wild-type and mutant sequences, respectively. The luciferase values were detected and analyzed. ${ }^{* *} \mathrm{P}<0.01$ vs. NC. VEGF, vascular endothelial growth factor; miR, microRNA; NC, normal control.

targeted regions in human and rat models. miRNA-23a is widely involved in the regulation of various physiological and pathological processes in the body, which plays an important role in the cellular differentiation (45-47). miRNA-23a has been shown to be involved in the formation of skeletal muscle, hematopoietic cell differentiation and bone metabolism (48). miRNA-23a expression levels in the skeletal muscle are relatively high, which has a negative correlation with the process of cell differentiation (49). miRNA-23a can form a positive feedback loop with Kruppel like factor 3 to promote the expression of $\beta$-like globulin genes involved in erythrocyte formation (50). Moreover, miRNA-23a could reduce the expression of alkaline phosphatase and osteocalcin genes, thus participating in the regulation of bone metabolism (51). Furthermore, miRNA-23a could promote the hypertrophy of cardiomyocytes, which is involved in the pathogenesis and development of atherosclerotic coronary heart diseases (52). These findings suggest that miRNA-23a may be involved in angiogenesis. The present study results indicated that miRNA-23a was significantly reduced in the serum and tear samples of patients with DR in comparison with controls. Similarly, the miRNA-24a expression levels in the serum and retina tissues of rat diabetic models were also significantly reduced. These results suggest that miRNA-23a may play an important role in the pathogenesis of DR. In the retina and serum samples from diabetic mice, abnormal expression levels of miRNA-20a-5p, miRNA-20a-3p, miRNA-20b, miRNA-10 6a-5p, miRNA-27a-5p, miRNA-27b-3p, miRNA-206-3p and miR-381-3p were observed. Moreover, the expression levels of VEGF, BDNF, PPAR- $\alpha$ and CREB1 have also been shown to be altered, indicating that there is a molecular interaction network (53).

To further investigate the molecular mechanism of miRNA-23a regulation of VEGF, in the present study hRMECs cells were cultured and transfected with agomiRNA-23a and the cell proliferation was analyzed. The results suggested 
A

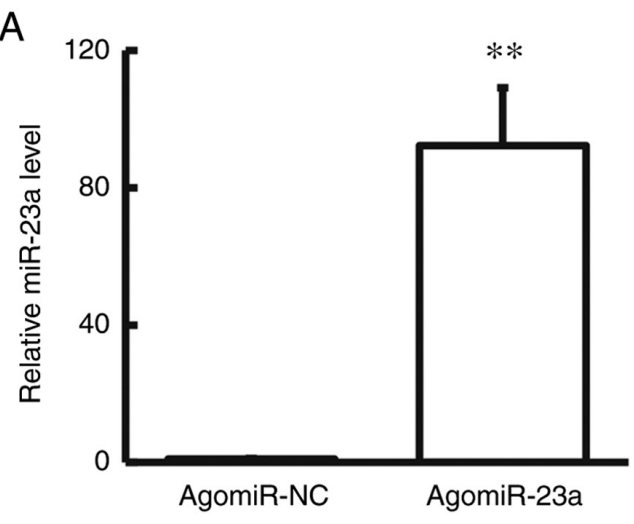

B

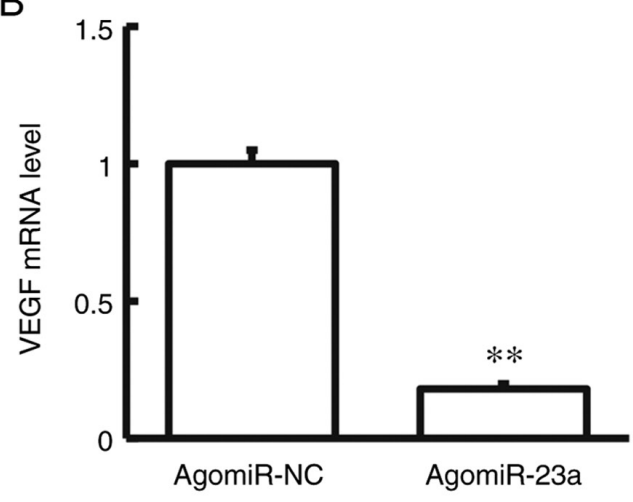

C

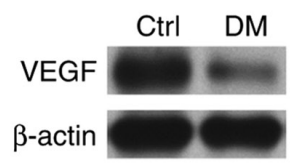

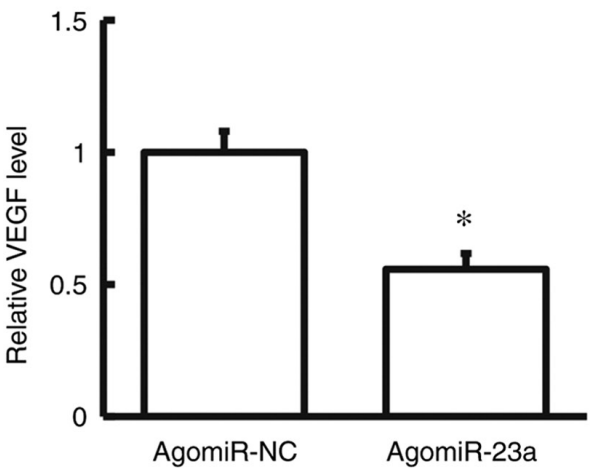

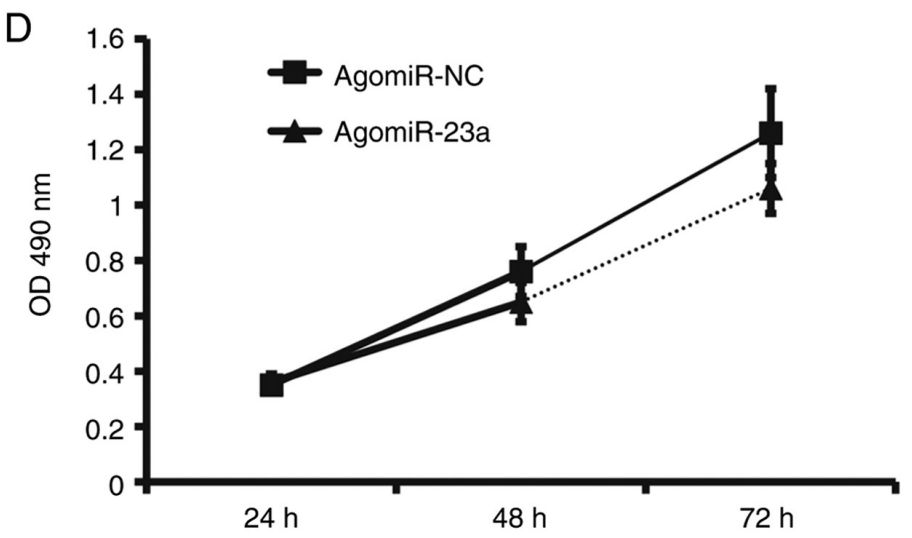

Figure 4. Effects of agomiRNA-23a transfection on hRMECs. hRMECs were transfected with agomiRNA-23a, and the expression levels of (A) miRNA-23a and (B) VEGF were determined with reverse transcription-quantitative-PCR. The VEGF protein expression levels were determined in the transfected hRMECs with (C) western blot analysis. (D) After transfection, cell proliferative ability was assessed with the MTT assay. ${ }^{*} \mathrm{P}<0.05,{ }^{* *} \mathrm{P}<0.01 \mathrm{vs}$. NC. miR, microRNA; hRMEC, human retinal microvascular endothelial cells; NC, normal control.

that miRNA-23a could slow down the cell proliferation of hRMECs. Moreover, the VEGF mRNA expression levels were reduced after miRNA-23a transfection. In order to confirm the direct binding of miRNA-23a and VEGF mRNA a dual luciferase reporter assay was performed. The results indicated that miRNA-23a could bind to the VEGF mRNA 3'-UTR seed region and regulate its expression levels.

There were several limitations to the present study. First, no longitudinal analysis of VEGF and miR-23 concentration with time was performed due to limited specimen availability, nor the association analysis had been performed. Additionally, it was not possible to assess all downstream targets of miR-23a, though these will be the subject of further research.

In conclusion, the results of the present study indicated that during DR pathogenesis miRNA-23a expression levels are reduced which increased the expression levels of VEGF and enhanced the proliferation of human retinal microvascular cells, promoting angiogenesis. These results suggest that miRNA-23a and VEGF play important roles in the pathogenesis and development of DR.

\section{Acknowledgements}

Not applicable.

\section{Funding}

This work was supported by grants from the National Natural Science Foundation of China (grant no. 81571383) and the China Postdoctoral Science Foundation (grant no. 2017M612870). 


\section{Availability of data and materials}

All data generated or analyzed during this study are included in this published article.

\section{Authors' contributions}

$\mathrm{LS}, \mathrm{XL}$ and $\mathrm{ZZ}$ contributed to the study design, performing the experiments, data collection and analysis and manuscript preparation. LS, XL and ZZ confirm the authenticity of all the raw data. All authors have read and approved the final manuscript.

\section{Ethics approval and consent to participate}

The study was approved by The Ethics Committee of the Third Affiliated Hospital of Jinzhou Medical University, and written informed consent was obtained from each subject.

\section{Patient consent for publication}

Not applicable.

\section{Competing interests}

The authors declare that they have no competing interests.

\section{References}

1. Cheung N, Mitchell P and Wong TY: Diabetic retinopathy. Lancet 376: 124-136, 2010.

2. Pan WW, Gardner TW and Harder JL: Integrative biology of diabetic retinal disease: Lessons from diabetic kidney disease. J Clin Med 10: 1254, 2021.

3. Sosna T: History of diagnosis and therapy of diabetic retinopathy. Vnitr Lek 62 (11 Suppl 4): S136-S141, 2016 (In Czech)

4. Liu Y and Wu N: Progress of nanotechnology in diabetic retinopathy treatment. Int J Nanomedicine 16: 1391-1403, 2021.

5. Inanc M, Tekin K, Kiziltoprak H, Ozalkak S, Doguizi S and Aycan Z: Changes in retinal microcirculation precede the clinical onset of diabetic retinopathy in children with type 1 diabetes mellitus. Am J Ophthalmol 207: 37-44, 2019.

6. Forst T, Weber MM, Mitry M, Müller L, Forst S, Tanis M, Pfützner A and Michelson G: Retinal microcirculation in type 1 diabetic patients with and without peripheral sensory neuropathy. J Diabetes Sci Technol 8: 356-361, 2014.

7. Vujosevic S, Toma C, Villani E, Gatti V, Brambilla M, Muraca A, Ponziani MC, Aimaretti G, Nuzzo A, Nucci P and De Cilla' S: Early detection of microvascular changes in patients with diabetes mellitus without and with diabetic retinopathy: Comparison between different swept-source OCT-A instruments. J Diabetes Res 2019: 2547216, 2019.

8. Heng LZ, Comyn O, Peto T, Tadros C, Ng E, Sivaprasad S and Hykin PG: Diabetic retinopathy: Pathogenesis, clinical grading, management and future developments. Diabet Med 30: 640-650, 2013.

9. Zheng Y, He M and Congdon N: The worldwide epidemic of diabetic retinopathy. Indian J Ophthalmol 60: 428-431, 2012.

10. Bromberg-White JL, Glazer L, Downer R, Furge K, Boguslawski E and Duesbery NS: Identification of VEGF-independent cytokines in proliferative diabetic retinopathy vitreous. Invest Ophthalmol Vis Sci 54: 6472-6480, 2013.

11. Koleva-Georgieva DN, Sivkova NP and Terzieva D: Serum inflammatory cytokines IL-1beta, IL-6, TNF-alpha and VEGF have influence on the development of diabetic retinopathy. Folia Med (Plovdiv) 53: 44-50, 2011.

12. Amadio M, Bucolo C, Leggio GM, Drago F, Govoni S and Pascale A: The PKCbeta/HuR/VEGF pathway in diabetic retinopathy. Biochem Pharmacol 80: 1230-1237, 2010.
13. The American Association of Neurological Surgeons (AANS), American Society of Neuroradiology (ASNR), Cardiovascular and Interventional Radiology Society of Europe (CIRSE), Canadian Interventional Radiology Association (CIRA), Congress of Neurological Surgeons (CNS), European Society of Minimally Invasive Neurological Therapy (ESMINT),European Society of Neuroradiology (ESNR), European Stroke Organization (ESO), Society for Cardiovascular Angiography and Interventions (SCAI), Society of Interventional Radiology (SIR), et al: Multisociety consensus quality improvement revised consensus statement for endovascular therapy of acute ischemic stroke. Int J Stroke 13: 612-632, 2018.

14. Platania CBM, Leggio GM, Drago F, Salomone S and Bucolo C: Computational systems biology approach to identify novel pharmacological targets for diabetic retinopathy. Biochem Pharmacol 158: 13-26, 2018.

15. Jain A, Saxena S, Khanna VK, Shukla RK and Meyer CH: Status of serum VEGF and ICAM-1 and its association with external limiting membrane and inner segment-outer segment junction disruption in type 2 diabetes mellitus. Mol Vis 19: 1760-1768, 2013.

16. Lu ES, Cui Y, Le R, Zhu Y, Wang JC, Laíns I, Katz R, Lu Y, Zeng R, Garg I, et al: Detection of neovascularisation in the vitreoretinal interface slab using Widefield swept-source optical coherence tomography angiography in diabetic retinopathy. $\mathrm{Br}$ J Ophthalmol 2020: 317983, 2020. (Epub ahead of print). doi: 10.1136/bjophthalmol-2020-317983.

17. Song S, Yu X, Zhang P and Dai H: Increased levels of cytokines in the aqueous humor correlate with the severity of diabetic retinopathy. J Diabetes Complications 34: 107641, 2020.

18. Behl T and Kotwani A: Exploring the various aspects of the pathological role of vascular endothelial growth factor (VEGF) in diabetic retinopathy. Pharmacol Res 99: 137-148, 2015.

19. Inoue $\mathrm{K}$ and Ogura A: Cloning mice: From aspects of donor cells. Tanpakushitsu Kakusan Koso 52 (Suppl 16): S2189-S2196, 2007 (In Japanese).

20. Williams AE, Moschos SA, Perry MM, Barnes PJ and Lindsay MA: Maternally imprinted microRNAs are differentially expressed during mouse and human lung development. Dev Dyn 236: 572-580, 2007.

21. Gomaa AR, Elsayed ET and Moftah RF: MicroRNA-200b expression in the vitreous humor of patients with proliferative diabetic retinopathy. Ophthalmic Res 58: 168-175, 2017.

22. Chen Q, Qiu F, Zhou K, Matlock HG, Takahashi Y, Rajala RVS, Yang Y, Moran E and Ma JX: Pathogenic role of microRNA-21 in diabetic retinopathy through downregulation of PPAR $\alpha$. Diabetes 66: 1671-1682, 2017.

23. Bao L, You B, Shi S, Shan Y, Zhang Q, Yue H, Zhang J, Zhang W, Shi Y, Liu Y, et al: Metastasis-associated miR-23a from nasopharyngeal carcinoma-derived exosomes mediates angiogenesis by repressing a novel target gene TSGA10. Oncogene 37: 2873-2889, 2018.

24. Hsu YL, Hung JY, Chang WA, Lin YS, Pan YC, Tsai PH, Wu CY and Kuo PL: Hypoxic lung cancer-secreted exosomal miR-23a increased angiogenesis and vascular permeability by targeting prolyl hydroxylase and tight junction protein $\mathrm{ZO}-1$. Oncogene 36: 4929-4942, 2017.

25. Di Y, Zhang D, Hu T and Li D: miR-23 Regulate the pathogenesis of patients with coronary artery disease. Int J Clin Exp Med 8: 11759-11769, 2015.

26. Zhang L, Lv Z, Xu J, Chen C, Ge Q, Li P, Wei D, Wu Z and Sun X: MicroRNA-134 inhibits osteosarcoma angiogenesis and proliferation by targeting the VEGFA/VEGFR1 pathway. FEBS J 285: 1359-1371, 2018.

27. Chen HX, Xu XX, Tan BZ, Zhang Z and Zhou XD: MicroRNA-29b inhibits angiogenesis by targeting VEGFA through the MAPK/ERK and PI3K/Akt signaling pathways in endometrial carcinoma. Cell Physiol Biochem 41: 933-946, 2017.

28. Vijan S: In the clinic. Type 2 diabetes. Ann Intern Med 162: ITC1-16, 2015.

29. Xu X: Ocular fundus disease in China: The current situation, progression, and issues to be resolved. Zhonghua Yan Ke Za Zhi 50: 801-803, 2014 (In Chinese).

30. Wellenberg A, Weides L, Kurzke J, Hennecke T, Bornhorst J, Crone B, Karst U, Brinkmann V, Fritz G and Honnen S: Use of C. elegans as a 3R-compliant in vivo model for the chemoprevention of cisplatin-induced neurotoxicity. Exp Neurol 341: 113705, 2021.

31. Ighodaro OM and Akinloye OA: Anti-diabetic potential of Sapium ellipticum (Hochst) Pax leaf extract in Streptozotocin(STZ)-induced diabetic Wistar rats. BMC Complement Altern Med 17: 525, 2017. 
32. Czescik A, Trzcinska A, Dunal-Szczepaniak M and Siennicka J: The use of real-time RT-PCR method for the determination of Toll-like genes expression at mRNA level. Med Dosw Mikrobiol 66: 17-22, 2014 (In Polish).

33. Betel D, Wilson M, Gabow A, Marks DS and Sander C: The microRNA.org resource: Targets and expression. Nucleic Acids Res 36: D149-D153, 2008.

34. Tang H, Zhang S, Huang C, Li K, Zhao Q and Li X: MiR-448-5p/VEGFA axis protects cardiomyocytes from hypoxia through regulating the FAS/FAS-L signaling pathway. Int Heart J 62: 647-657, 2021.

35. Yan Z, Hong S, Song Y and Bi M: microR-4449 Promotes colorectal cancer cell proliferation via regulation of SOCS3 and activation of STAT3 signaling. Cancer Manag Res 13:3029-3039, 2021.

36. Wu X,Lei J,Zhou B, Sun Q, Gao Y, Shi F and Yang W: MiR-628-5p inhibits cervical carcinoma proliferation and promotes apoptosis by targeting VEGF. Am J Med Sci 361: 499-508, 2021.

37. Nishinaka A, Nakamura S, Tanaka M, Masuda T, Inoue Y, Yamamoto T, Imai T, Hidaka Y, Shimazawa M and Hara $\mathrm{H}$ : Excess adiponectin in eyes with progressive ocular vascular diseases. FASEB J 35: e21313, 2021.

38. Zhang W, Liu H, Al-Shabrawey M, Caldwell RW and Caldwell RB: Inflammation and diabetic retinal microvascular complications. J Cardiovasc Dis Res 2: 96-103, 2011.

39. Kovacs K, Marra KV, Yu G, Wagley S, Ma J, Teague GC, Nandakumar N, Lashkari K and Arroyo JG: Angiogenic and inflammatory vitreous biomarkers associated with increasing levels of retinal ischemia. Invest Ophthalmol Vis Sci 56: 6523-6530, 2015.

40. Umeda N, Ozaki H, Hayashi H, Kondo H, Uchida H and Oshima K: Non-paralleled increase of hepatocyte growth factor and vascular endothelial growth factor in the eyes with angiogenic and nonangiogenic fibroproliferation. Ophthalmic Res 34: 43-47, 2002.

41. Calvo PM, de la Cruz RR and Pastor AM: Synaptic loss and firing alterations in Axotomized Motoneurons are restored by vascular endothelial growth factor (VEGF) and VEGF-B. Exp Neurol 304: 67-81, 2018.

42. Chernykh VV, Varvarinsky EV, Smirnov EV, Chernykh DV and Trunov AN: Proliferative and inflammatory factors in the vitreous of patients with proliferative diabetic retinopathy. Indian J Ophthalmol 63: 33-36, 2015.

43. Kianersi F, Ghanbari H, Naderi Beni Z and Naderi Beni A: Intravitreal vascular endothelial growth factor (VEGF) inhibitor injection in patient during pregnancy. J Drug Assessment 10: 7-9, 2021.

44. Ang WJ, Zunaina E, Norfadzillah AJ, Raja-Norliza RO, Julieana M, Ab-Hamid SA and Mahaneem M: Evaluation of vascular endothelial growth factor levels in tears and serum among diabetic patients. PLoS One 14: e0221481, 2019.
45. Wu F, Wang F, Yang Q, Zhang Y, Cai K, Liu L, Li S, Zheng Y, Zhang J, Gui Y, et al: Upregulation of miRNA-23a-3p rescues high glucose-induced cell apoptosis and proliferation inhibition in cardiomyocytes. In Vitro Cell Dev Biol Anim 56: 866-877, 2020.

46. Huang Y, Huang J, Qi R, Wang Q, Wu Y and Wang J: Effects of MicroRNA-23a on differentiation and gene expression profiles in 3T3-L1 adipocytes. Genes (Basel) 7: 92, 2016.

47. Shen L, Chen L, Zhang S, Zhang Y, Wang J and Zhu L: MicroRNA-23a reduces slow myosin heavy chain isoforms composition through myocyte enhancer factor 2C (MEF2C) and potentially influences meat quality. Meat Sci 116: 201-206, 2016.

48. Xu Y, Jiang Y, Jia B, Wang Y and Li T: Icariin stimulates osteogenesis and suppresses adipogenesis of human bone mesenchymal stem cells via miR-23a-mediated activation of the $\mathrm{Wnt} / \beta$-catenin signaling pathway. Phytomedicine 85: 153485, 2021.

49. Hernandez-Torres F, Aranega AE and Franco D: Identification of regulatory elements directing miR-23a-miR-27a-miR-24-2 transcriptional regulation in response to muscle hypertrophic stimuli. Biochim Biophys Acta 1839: 885-897, 2014.

50. Ma Y, Wang B, Jiang F, Wang D, Liu H, Yan Y, Dong H, Wang F, Gong B, Zhu Y, et al: A feedback loop consisting of microRNA $23 \mathrm{a} / 27 \mathrm{a}$ and the $\beta$-like globin suppressors KLF3 and SP1 regulates globin gene expression. Mol Cell Biol 33: 3994-4007, 2013.

51. Zhang Y, Xie RL, Croce CM, Stein JL, Lian JB, van Wijnen AJ and Stein GS: A program of microRNAs controls osteogenic lineage progression by targeting transcription factor Runx2. Proc Natl Acad Sci USA 108: 9863-9868, 2011.

52. Han H, Qu G, Han C, Wang Y, Sun T, Li F, Wang J and Luo S: MiR-34a, miR-21 and miR-23a as potential biomarkers for coronary artery disease: A pilot microarray study and confirmation in a 32 patient cohort. Exp Mol Med 47: e138, 2015.

53. Platania CBM, Maisto R, Trotta MC, D'Amico M, Rossi S, Gesualdo C, D'Amico G, Balta C, Herman H, Hermenean A, et al: Retinal and circulating miRNA expression patterns in diabetic retinopathy: An in silico and in vivo approach. Br J Pharmacol 176: 2179-2194, 2019.

This work is licensed under a Creative Commons Attribution-NonCommercial-NoDerivatives 4.0 International (CC BY-NC-ND 4.0) License. 